\title{
Clinical, dermoscopic, and histologic aspects of two cases of cutaneous focal mucinosis*
}

\author{
Giovanni Biondo ${ }^{1}$, Simona Sola², Carlotta Pastorino ${ }^{3}$, Cesare Massone ${ }^{3}$
}

DOI: http:/ / dx.doi.org/10.1590/abd1806-4841.20198381

\begin{abstract}
Cutaneous mucinoses are a complex and diverse group of connective tissue disorders characterized by the accumulation of mucin and/or glycosaminoglycan in the skin and adnexa. Cutaneous focal mucinosis appears as a solitary, asymptomatic, skin-colored to white papule, nodule, or plaque located anywhere on the body or in the oral cavity. It presents mainly in adults and is characterized on histopathology by mucin throughout the upper and mid dermis. We describe the dermoscopy of two cases of cutaneous focal mucinosis. Both lesions presented a nonspecific homogenous whitish pattern; the first case also exhibited a sharply demarcated yellow border.
\end{abstract}

Keywords: Dermoscopy; Pathology; Skin abnormalities

\section{INTRODUCTION}

Cutaneous mucinoses are a complex and diverse group of connective tissue disorders characterized by the accumulation of mucin and/or glycosaminoglycan in the skin and adnexa. ${ }^{1}$ It is unclear why mucin accumulates in the skin. ${ }^{2}$ Cutaneous focal mucinosis (CFM) was first described by Johnson and Helwig in $1966 .{ }^{3}$ It typically appears as a solitary, asymptomatic, skin-colored to white papule, nodule, or plaque located anywhere on the body or in the oral cavity. CFM is classified as primary cutaneous mucinosis, subtype degenerative-inflammatory. ${ }^{4}$ CFM presents mainly in adults and is characterized on histopathology by mucin throughout the upper and mid dermis, except the subcutaneous fat; cleftlike spaces and spindle-shaped or stellate fibroblasts are present. ${ }^{3,5}$ CFM results from a muciparous reaction of the connective tissue to nonspecific stimuli and should be distinguished histopathologically from digital mucous cyst as well as angiomyxoma, fibromyxoma, and myxofibroma.

\section{CASE REPORT}

We report the dermoscopic findings from two cases of CFM. A 55-year-old man (patient \#1) presented with a longstanding skin-colored, asymptomatic nodule, $8 \mathrm{~mm}$ in diameter, on the back (Figure 1). A 61-year-old woman (patient \#2) showed a small, 6 $\mathrm{mm}$ in diameter, skin-colored asymptomatic nodule on the left forearm (Figure 2). Upon dermoscopy, both lesions presented a nonspecific homogenous whitish pattern; the first case also exhibited a sharply demarcated yellow border (Figure 3 and 4$).{ }^{6}$ Both lesions were excised and on microscopic examination showed a myxomatous stroma in the dermis, atrophy of the epidermis, scattered spindle-shaped and stellate fibroblasts, and collagen-fiber dissociation in the dermis (cleft-like spaces). The subcutis was not involved. Alcian blue staining showed abundant deposits of mucin in both cases (Figure 5, patient \#1). Melan-A stain revealed a decreased number of melanocytes in the epidermis above the mucin deposits (Figure 6, patient \#2).

\footnotetext{
Received 20 April 2018.

Accepted 06 September 2018

* Work performed at the Dermatology Unit, Galliera Hospital, Genova, Italy Financial support: None.

Conflict of interest: None.

Department of Dermatology, P. Giaccone Hospital, University of Palermo, Palermo, Italy.
Surgical Pathology Unit, Galliera Hospital, Genova, Italy.

Dermatology Unit, Galliera Hospital, Genova, Italy.
}

MAILING AdDREsS:

Giovanni Biondo

E-mail: dott.giovannibiondo@gmail.com

C2019 by Anais Brasileiros de Dermatologia 


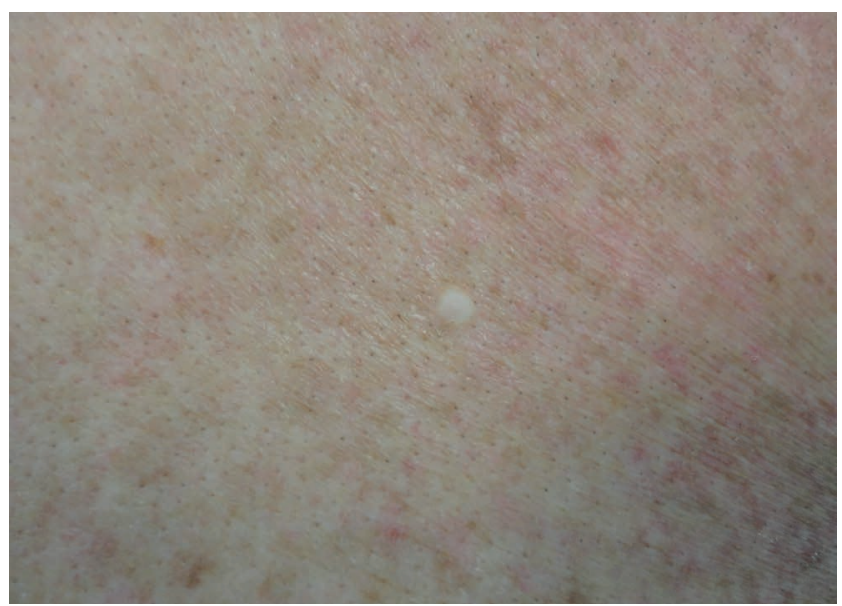

FIGURE 1: Long-standing skin-colored 8mm-diameter asymptomatic nodule on the back (patient \#1)

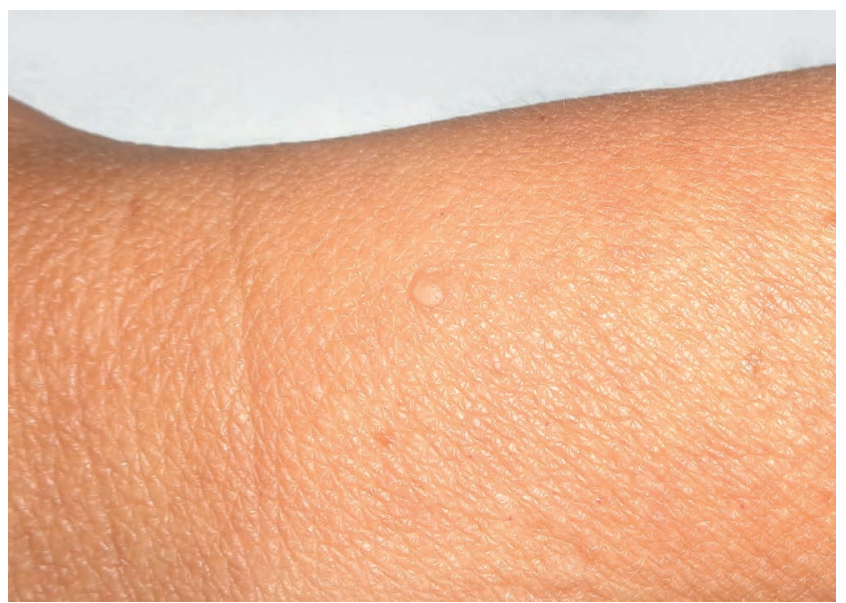

FigURE 2: A small, $6 \mathrm{~mm}$-diameter skin-colored asymptomatic nodule on the left forearm (patient \#2)

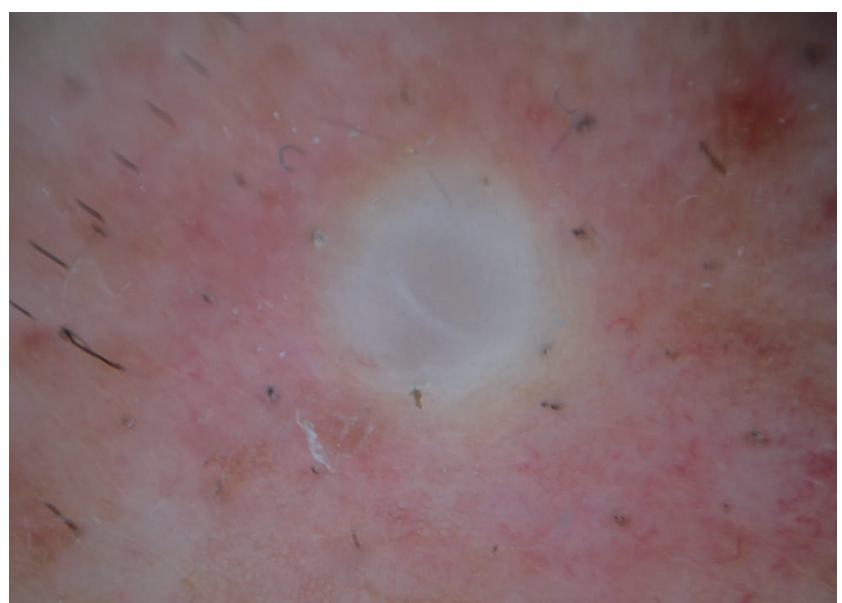

FIGURE 3: Nonspecific homogenous whitish pattern and a sharply demarcated yellow border on dermoscopy (patient \#1)

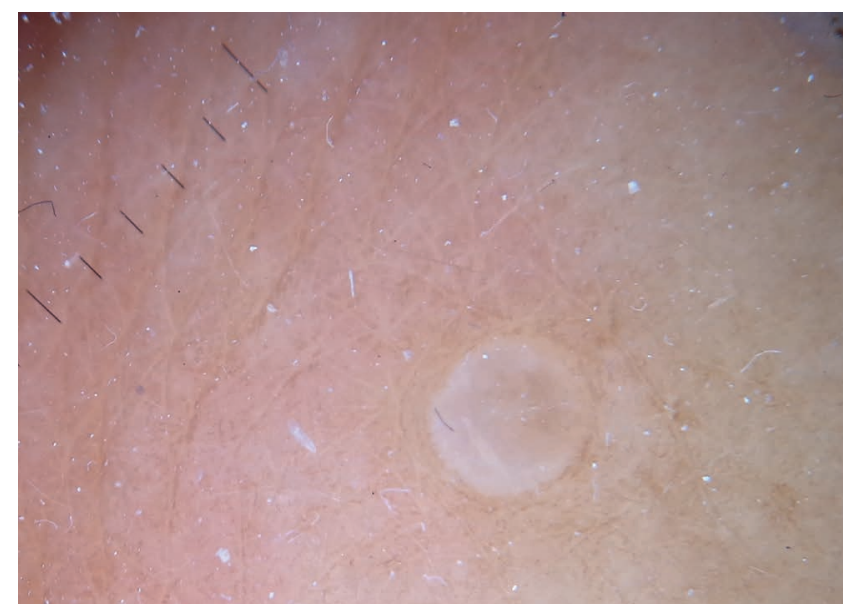

FIGURE 4: Nonspecific homogenous whitish pattern on dermoscopy (patient \#2)

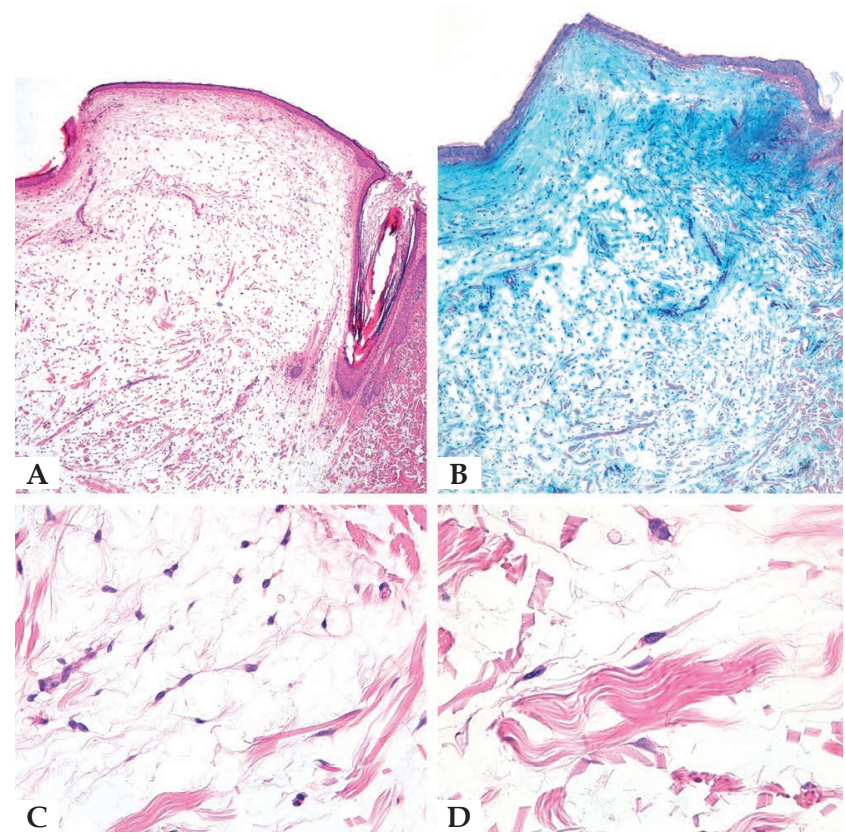

Figure 5: A. Microscopic examination showed myxomatous stroma in the dermis, atrophy of the epidermis, scattered spindle-shaped fibroblasts, and dissociation of collagen fibers in the dermis (cleftlike spaces) (patient \#1) (Hematoxylin \& eosin, x20); B. Alcian blue staining showed abundant mucin deposits (patient \#1)(x20); C and D. Higher magnifications show spindle and stellate fibroblasts in a myxoid area (patient \#1) (Hematoxylin \& eosin, 5C x200; 5D x 400)

\section{DISCUSSION}

CFM is a benign, single, solitary cutaneous mucinosis with a broad spectrum of clinical presentation, usually less than $1 \mathrm{~cm}$ in diameter, and unassociated with mucinosis-related systemic diseases. CFM can be removed by simple surgical excision. Average age at presentation varies from 38 to 50 years, ${ }^{3}$ and incidence appears to be higher in males than in females. ${ }^{3,7}$ Histopathology assists differential 


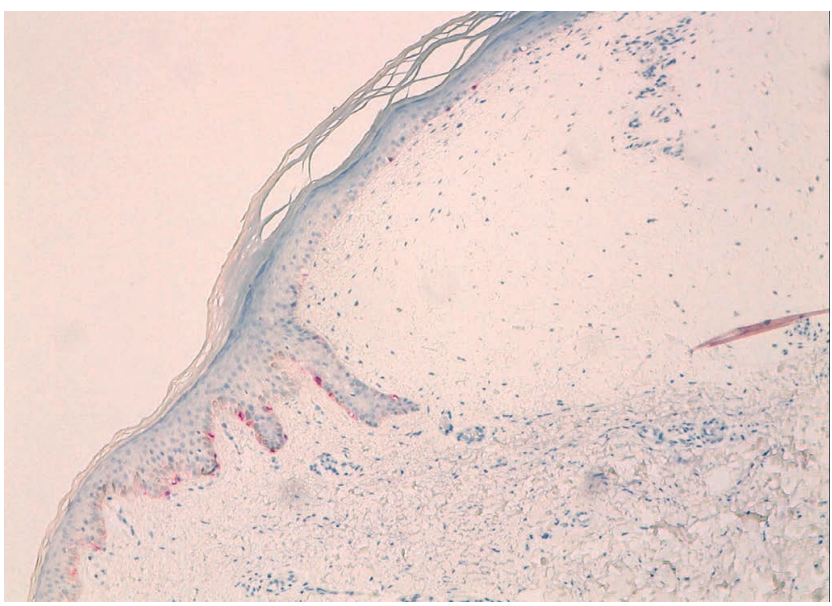

FIGURE 6: Melan-A staining shows reduced melanocytes in the epidermis above the mucin deposition in the dermis (patient \#2) $(x 40)$

diagnosis by distinguishing CFM from an initial clinical impression of myxoma, leukemia cutis, epidermal inclusion cyst, soft fibroma, melanocytic nevus, skin appendage tumor, Rosai-Dorfman disease, eccrine poroma, nodular hidradenoma, follicular cyst, acrochordon, or hidroacanthoma simplex. ${ }^{7}$ The finding of a dome-shaped space oriented horizontally (mucin) in the upper part of the dermis below a thinned epidermis surrounded by compressed fibrous tissue is a sign of CFM.
Our patients' lesions presented a nonspecific homogenous whitish pattern, and one also exhibited a sharply demarcated yellow border. The white appearance may be partly explained by the decreased number of melanocytes in the epidermidis above the mucin deposits in the dermis (Figure 6). Another possible explanation is that mucin has birefringent properties like collagen bundles that result in rapid randomization and increased backscatter of polarized light, which makes collagen appear bright white under polarized dermoscopy, as postulated in crystalline/chrysalis structures. ${ }^{8}$

Although the homogeneous whitish pattern and yellow border are not unique to CFM, we believe that dermoscopy can add useful information to naked-eye examination. A homogeneous pattern has been described in several entities. In the dermoscopy of dermatofibroma, a homogeneous whitish center and a peripheral pigmentary network is a common pattern. ${ }^{9}$ Extensive regression in pigmented skin lesions can show a homogeneous whitish pattern, but the presence of peppering can help distinguish it from CFM.

Fibrotic lesions such as scars, atrophie blanche, and calcinosis cutis also present a homogeneous whitish pattern on dermoscopy, but the scars are linear, the lesions are multiple in calcinosis cutis, and atrophie blanche is flat with post-inflammatory hyperpigmentation on the periphery.

It is important to recognize CFM in order to avoid misdiagnosis as common lesions such as molluscum, warts, milia, and cysts. To the best of our knowledge, ours is the first report on the dermoscopy of CFM. $\square$

\section{REFERENCES}

1. Rongioletti F, Rebora A. The new cutaneous mucinosis: a review with an up-to-date classification of cutaneous mucinosis. J Am Acad Dermatol. 1991;24:265-70.

2. Edward M, Fitzgerald L, Thind C, Leman J, Burden AD. Cutaneous mucinosis associated with dermatomyositis and nephrogenic fibrosing dermopathy: fibroblast hyaluronan synthesis and effect of patient serum. $\mathrm{Br} J$ Dermatol. 2007;156:473-9.

3. Johnson WC, Helwig EB. Cutaneous focal mucinosis. A clinicopathological and histochemical study. Arch Dermatol. 1966;93:13-20.

4. Rongioletti F, Amantea A, Balus L, Rebora A. Cutaneous focal mucinosis associated with reticular erythematous mucinosis and scleromyxedema. J Am Acad Dermatol. 1991;24:656-7.

5. Nebrida ML, Tay YK.. Cutaneous focal mucinosis: a case report. Pediatr Dermatol. 2002:19:33-5.
6. Kittler H, Marghoob AA, Argenziano G, Carrera C, Curiel-Lewandrowski C, Hofmann-Wellenhof $\mathrm{R}$, et al. Standardization of terminology in dermoscopy/ dermatoscopy: Result of the third consensus conference of the International Society of Dermoscopy. J Am Acad Dermatol. 2016;74:1093-106.

7. Kuo KL, Lee LY, Kuo TT. Solitary cutaneous focal mucinosis: a clinicopathological study of 11 cases of soft fibroma-like cutaneous mucinous lesions. J Dermatol. 2017;44:335-8.

8. Pan Y, Gareau DS, Scope A, Rajadhyaksha M, Mullani NA, Marghoob AA. Polarized and nonpolarized dermoscopy: the explanation for the observed differences. Arch Dermatol. 2008;144:828-9.

9. Zaballos P, Puig S, Llambrich A, Malvehy J. Dermoscopy of Dermatofibromas A prospective Morphological Study of 412 Cases. Arch Dermatol. 2008;144:75-83.

\section{AUTHORS'CONTRIBUTIONS}

\section{Giovanni Biondo $\quad$ (iD) ORCID 0000-0002-9710-8223}

Approval of the final version of the manuscript; Conception and planning of the study; Elaboration and writing of the manuscript; Obtaining, analyzing and interpreting the data; Effective participation in research orientation; Intellectual participation in propaedeutic and/or therapeutic conduct of the cases studied; Critical review of the literature; Critical review of the manuscript

Simona Sola $\quad$ (iD) ORCID 0000-0001-5305-7949

Approval of the final version of the manuscript; Obtaining, analyzing and interpreting the data; Intellectual participation in propaedeutic and/or therapeutic conduct of the cases studied; Critical review of the literature

\section{Carlotta Pastorino \\ ORCID 0000-0003-1644-4303}

Approval of the final version of the manuscript; Intellectual participation in propaedeutic and/or therapeutic conduct of the cases studied
Cesare Massone
iD ORCID 0000-0002-0996-643X

Approval of the final version of the manuscript; Elaboration and writing of the manuscript; Obtaining, analyzing and interpreting the data; Effective participation in research orientation; Intellectual participation in propaedeutic and/or therapeutic conduct of the cases studied; Critical review of the literature; Critical review of the manuscript

How to cite this article: Biondo G, Sola S, Pastorino C, Massone C. Clinical, dermoscopic, and histologic aspects of two cases of cutaneous focal mucinosis. An Bras Dermatol. 2019;94(3):334-6. 